\title{
Appliance of domiciliary nutrition instruction method for the elderly people requiring long- term care in Japan
}

\author{
Koko Rusu-Udagawa ${ }^{1 *}$, Toshikazu Suzuki ${ }^{2}$, Yukie Yanagisawa ${ }^{2}$ \\ From Metabolism, diet and disease \\ Washington, DC, USA. 29-31 May 2012
}

\section{Background}

In Japan, elderly people aged 65 and over account for more than one fifth of the nation's population. To add to this, the number of aged households is increasing year by year. Because elderly people are preferential to have tender and non-fatty foods in general, they are prone to biased nutrition status. According to the result of a National Health and Nutrition Survey, the low weight male who aged 60-69 and over percentage has increased from $7.2 \%$ (1987) to $12.3 \%$ in 2009 . Also, female is $18.6 \%$ to $22.3 \%$. Therefore both male and female has increased about $5 \%$. lt is predicted that nutritional management for elderly person requiring long-term care but living in their home may become difficult. Establishing the novel nutritional guidance for such elderly person with their favor is one of the most important challenges for clinical dietitian in Japan. In this study, we carried out two questionnaire surveys, in order to understand nutritional status of elderly people requiring long-term care but living in their home and find out dietary preference of elderly people.

\section{Materials and methods}

In order to grasp the present condition of care of elderly people requiring long-term care, we performed the questionnaire by mail to 197 nursing-care-services entrepreneurs in the part of Tokyo. In order to be and to grasp the present condition of house elderly people's meal, both the part of Tokyo and one of city in the Chiba used semiquantitative food frequency questionnaire (FFQ) method which set the elderly people aged 65 and over as the 147person object. Also, we performed the questionnaire about the cooking method also went simultaneously.

'Graduate School of Human Ecology, Wayo Women's University, Ichikawa,

Chiba 272-8533, Japan

Full list of author information is available at the end of the article

\section{Results}

According to the nursing-care-services entrepreneur's questionnaire, there was a problem as decrease of dietary intake which is $78.0 \%$, decrease of weight of $39.6 \%$, malnutrition of $54.9 \%$. According to FFQ, it was characteristic that people intake of fish and shellfishes more than meat, also soft food, such as a sweet roll. Moreover, in investigation of the cooking method, there was much ingestion of the sliced raw fish, and it was simple cooking method, people would rather cook as bake.

\section{Conclusions}

When the decrease of dietary intake is seen in connection with aging, it is necessary to recommend food with high nutrient density. Moreover, it is also important to propose the meal content which took dysphagia function into consideration enough. It is important that dietitian recommends simple recipes using microwave oven to elderly people and their family, rather than telling recipes for elaborate meals.

\section{Author details}

'Graduate School of Human Ecology, Wayo Women's University, Ichikawa, Chiba 272-8533, Japan. ${ }^{2}$ Department of Health and Nutrition, Wayo Women's University, Ichikawa, Chiba 272-8533, Japan.

Published: 1 June 2012

doi:10.1186/1753-6561-6-S3-P50

Cite this article as: Rusu-Udagawa et al:: Appliance of domiciliary nutrition instruction method for the elderly people requiring long-term care in Japan. BMC Proceedings 2012 6(Suppl 3):P50. 